\title{
Article
}

\section{Research Towards High Speed Freeforming}

Brooks, Hadley Laurence, Piorkowski, Barry Andrew and Lupeanu, Mihaela

Available at http://clok.uclan.ac.uk/7353/

Brooks, Hadley Laurence ORCID: 0000-0001-9289-5291, Piorkowski, Barry Andrew and Lupeanu, Mihaela (2013) Research Towards High Speed

Freeforming. International Journal of Rapid Manufacturing, 3 (2/3). pp. 154171. ISSN 1757-8817

It is advisable to refer to the publisher's version if you intend to cite from the work. http://dx.doi.org/10.1504/JJRAPIDM.2013.053686

For more information about UCLan's research in this area go to http://www.uclan.ac.uk/researchgroups/ and search for <name of research Group>.

For information about Research generally at UCLan please go to http://www.uclan.ac.uk/research/

All outputs in CLoK are protected by Intellectual Property Rights law, including Copyright law. Copyright, IPR and Moral Rights for the works on this site are retained by the individual authors and/or other copyright owners. Terms and conditions for use of this material are defined in the policies page.

\section{CLoK}

Central Lancashire online Knowledge www.clok.uclan.ac.uk

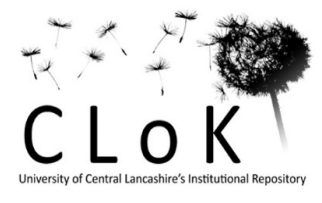


Abstract:

Additive manufacturing (AM) methods are currently utilised for the manufacture of prototypes and low volume, high cost parts. This is because in most cases the high material costs and low volumetric deposition rates of AM parts result in higher per part cost than traditional manufacturing methods. This paper brings together recent research aimed at improving the economics of $A M$, in particular Extrusion Freeforming (EF).

A new class of machine is described called High Speed Additive Manufacturing (HSAM) in which software, hardware and materials advances are aggregated. HSAM could be cost competitive with injection moulding for medium sized medium quantity parts. A general outline for a HSAM machine and supply chain is provided along with future required research.

Keywords: Additive Manufacturing, Rapid Manufacturing, Fused Deposition Modelling, Medium Volume Production, High Speed Extrusion Freeforming.

1. Introduction:

Current consumer trends indicate a desire for more customised and one of a kind goods. In addition to this the current emphasis in product development has been to reduce the product development cycle in order to compete in the global market (Bernard and Fischer, 2002). Rapid prototyping (RP) technologies, developed in the 1980's, have helped to speed product development cycles by providing prototypes for concept validation and functional testing. As these RP technologies have continued to improve the focus has shifted from prototyping to the rapid manufacture of end use parts. Now these technologies are being accepted as mainstream manufacturing methods in their own right and the term additive manufacturing (AM) is used to signify the shift from prototyping to proper manufacturing. The constant development of AM technologies and the increasing consumer demand for custom products has resulted in the increased use of AM for retail goods (Brooks et al, 2012).

The typical AM process involves building parts directly from computer models in a layer wise fashion. As AM does not require the use of expensive tooling this can lead to large reductions in the cost of complex low volume components or assemblies. AM is also used for high value added parts in the medical, aerospace and jewellery sectors where the design freedoms allowed by AM enable the manufacture of complex, high specification or highly functional parts (Lupeanu et al, 2012).

While AM has been touted as a suitable candidate for mass customisation, a number of barriers have prevented the wide spread adoption of this technology. The layer based manufacturing methods lead to low volumetric production rates compared with conventional mass production methods such as injection moulding (Gibson et al., 2010). A number of studies have been carried out comparing the per part cost of the various AM technologies against each other and against conventional manufacturing technologies, such as machining or injection moulding. One such study by Hopkinson and Dickens (2002) compared the feasibility of manufacturing a small plastic lever using Stereolithography (SL), Fused Deposition Modelling (FDM), Laser Sintering (LS) and injection moulding over a range of production quantities. The per part cost of AM components is only slightly affected by the quantity, however the per part cost for injection moulding is expensive for low quantities and inexpensive for high volume quantities as the cost of the tooling is spread over the parts. SL was found to be the most economical AM technology, being cost competitive with injection moulding up to 14,000 parts. SL and FDM were approximately twice as expensive as SL and was only competitive with injection moulding quantities up to 7,000 parts. When the same analysis was done with a medium sized plastic part the cross over point between AM and injection moulding occurred at much lower production volumes. This shows the volumetric deposition rate and material cost of AM technologies is a real limitation for the wide spread use of AM for medium volume production. Of course this comparison does not take into account the quality of the different parts, nor does it recognise that for AM each of the parts could be geometrically different at no extra cost.

\subsection{Fused deposition modelling and Extrusion Freeforming}

FDM is one of the most widely used commercial extrusion methods, and remains one of the most versatile. Extrusion Freeforming (EF) is the term that will be used to describe extrusion technologies that differ from FDM, which is trademarked by Stratasys. EF parts are built by depositing a small bead of softened polymer through an 
extrusion head onto a work platform. The nozzle (or platform) moves via computer control to lay down a pattern on the platform. Complete parts are built by laying down successive patterns one layer at a time. Since the expiration of the original patent for FDM (Crump, 1989), sales of low cost extrusion based machines have increased dramatically (Wohlers, 2012). This has also lead to a corresponding increase in the number of vendors providing filament materials and a consequent drop in material prices. Due to the fact many different materials can be extruded through a nozzle, extrusion based AM provides greater flexibility in producing multi-material parts and assemblies than powder based methods. However, FDM has a number of short comings when compared with other AM technologies. In particular, the build speed, strength and surface quality of FDM parts is inferior to LS parts (Brajlih et al, 2011).

\subsection{What is High Speed AM?}

Most current AM technologies are still based on concepts originating in the 80's and 90's for developing prototype parts, such as, uniform layer thicknesses, single point of material addition and homogenous materials. If AM is to reach its potential as a credible manufacturing technology for medium to large volume production, a paradigm shift is needed in the way these machines are designed and operated. A new breed of AM machines is required.

Development of this new breed of machines and associated software has already begun, although many of these advances are yet to be commercialised and much more research is needed. To differentiate the abilities of these new machines the authors suggest the term 'High Speed Additive Manufacturing' or HSAM to refer to AM machines capable of medium to high volume production of small to medium sized parts with consumer required quality. In this case medium volume is 1,000 to 10,000 parts. While medium size refers to parts approximately $8 x$ $10^{6} \mathrm{~mm}^{3}$ or $200 \mathrm{~mm} \times 200 \mathrm{~mm} \times 200 \mathrm{~mm}$. It is easy to imagine the benefits of a HSAM production facility which can simultaneously manufacture a variety of multi-material assemblies with a minimum number of operations and zero downtime for product change over. Designs from anywhere could be uploaded to a cloud and printed out near their point of use. HSAM production facilities would require minimal manual human input. Therefore there would be less advantage for HSAM facilities to be located in countries with low manual labour rates, and products could be made closer to where they will be used. Local manufacturing significantly reduces the carbon footprint caused by the mass transit of consumer goods.

EF is capable of building parts and assemblies with multiple materials, ranging from biological tissue to fibre reinforced composites and electronics. This paper will focus on current and future research aimed at developing EF into HSEF. The different areas of development towards HSEF can be split into three broad categories; software, hardware and materials. It should be noted however that many of the ideas explained in this paper are applicable to AM technologies in general.

\section{HSEF software developments}

Within AM the majority of software developments focus on slicing algorithms, tool path planning and part orientation/nesting. Slicing algorithms are used to determine how the STL file is sliced and how thick the slices are. In EF, tool path planning is then used to determine the extrusion path and to calculate nozzle speeds and extruder feed rates. Nesting algorithms are not as important in EF as they are for powder based methods and so will be omitted here. Conventional EF uses constant layer height slicing, homogeneous build materials and three axis control. The following sections outline recent research that could progress EF towards HSEF.

\subsection{Adaptive layer slicing}

Conventional FDM uses constant layer thicknesses, typically between 0.1 and $0.4 \mathrm{~mm}$ as shown in Figure 1a. Using smaller layers increases the part accuracy and reduces the surface roughness but also increases the build time. Variable layer thickness like that shown in Figure $1 \mathrm{~b}$ can be used to increase the build speed while maintaining high levels of accuracy. Most slicing algorithms aim to reduce or limit the cusp height of the hypothetical rectangular layer profiles.

Pandy et al, (2003) provide a good review of the different adaptive slicing procedures whist in another paper (Pandey et al, 2003) they introduce an improved slicing algorithm by factoring in a more realistic parabolic profile. Tyberg and Bohn, (1999) introduced local adaptive slicing where the parts or part features are separated and sliced independently. This allows different parts or features on the same build platform to be built with their own optimal layer slicing. 
A particularly interesting slicing algorithm was developed by Sabourin et al, (1997) known as accurate exterior, fast interior layered manufacturing. This slicing method allows thinner layers on the outside of the part with thicker layers to be deposited on the inside as shown in Figure 2. The thicker layers must be multiples of the outer layer thicknesses. This slicing procedure is most beneficial for large solid objects.

The claimed increase in build speed varies between the different slicing procedures and also between different part geometries. However reduction in build time when compared with conventional FDM, for a variety of parts has been shown to fall between 42 and $73 \%$. This advancement alone could move EF a long way towards economic parity with injection moulding for solid medium sized, medium volume parts. While these slicing procedures were developed primarily for EF they are applicable to most AM technologies.

Software development is still required to implement these slicing procedures with easy to use GUls. Extensive testing is also required to assess build issues such as heat build-up. With higher deposition rates there will be less time for the new layers to dissipate heat. This may cause a lack of rigidity in the older layers which are supporting the new layers. Other issues include maintaining geometric control of high volume roads or filaments and designing extruders capable of deposition over a wider volumetric range.

\subsection{Multiple material tool path generation}

When printing multiple material parts with multiple extrusion heads, extruder collisions must be avoided. Zhu and $\mathrm{Yu},(2002)$ describe a method of generating tool paths for multiple material assemblies or parts with discrete regions of materials. Figure 3 shows an example of a multi material assembly. The exploded view depicts the shapes of each material separately.

The tool path generation is a dexel based spatio-temporal modelling approach which is computationally efficient. The dexels (depth pixels) are equally spaced parallel lines that intersect horizontal planes of the model and provide a foundation for the tool paths (figure 4).

The spatio-temporal modelling of the tool paths of each layer can be visualised by considering time as an extra spatial dimension. This converts the 2D tool paths into 3D surfaces. Extruder collisions are then easily detected by analysing whether the 3D surfaces intersect in space-time. This method can be extended to a hyper-solid model in space-time to detect collisions for the whole part. This method makes it possible to generate tool paths that allow separate deposition heads to add different materials simultaneously. Hardware considerations such as the geometry of the deposition heads and axis will need to be considered, however for large multi-material parts, or the simultaneous building of a batch of parts with different materials, this tool path generation method could reduce build time significantly.

Not only is it possible to print parts with multiple discrete materials but it is also possible to print parts with graded materials as shown in figure 5. Parts with functionally graded materials result in parts that have spatially varied mechanical, thermal and electrical properties. The potential applications for heterogeneous materials in manufactured parts are significant.

Zhou, (2004) developed an adaptive slicing algorithm with the additional ability of generating tool paths for functionally graded materials. Zhou's method for adaptively slicing the solid model is similar to those mentioned earlier. However, additional steps are required to discretise the continuous material distribution into stepwise subregions. The minimum possible variation in adjacent material sub-regions is defined as the material resolution. The boundaries of the material sub-regions are analogous to contour lines on a map. Figure 6 below shows how the upper layer cross section in figure 4 will be discretised to account for the material resolution.

When building multi-material or mixed material parts it is important to ensure the individual materials are compatible and can form strong bonds. Further research is required to establish ways in which AM composite materials work together. This is an issue not only relating to the chemical or molecular bonds but also to how the mechanical, thermal and electrical loads will be distributed. In some cases coupling agents may be needed, for example to join hydrophobic and hydrophilic materials. In extreme cases where completely incompatible materials are to be joined, the material may need to be geometrically coupled. This could be done by designing in $3 \mathrm{D}$ dovetail like features into the mesostructure of the material interfaces. The recyclability of multi-material parts also needs to be considered. 


\subsection{Design for multi-material parts}

One of the main challenges facing the design, and ultimately the use of multi-material parts is not the hardware but the design methods used by the engineers themselves. The ability to build multi-material parts increases the design solution space significantly. New software is needed to help designers achieve optimum design solutions within this much larger space.

Hiller and Lipson, (2009) presented an evolutionary design algorithm for 3D multi-material structures. Evolutionary algorithms are well suited for the optimisation of large solution spaces where competing goals are frequent. As the quantity and complexity of customised products increases, design automation will become ever more essential.

\subsection{Curved layer FDM and 5 axis EF}

For certain part geometries, the flat build layers of traditional AM parts is very inefficient. A good example of this is thin walled curved shell-like structures. In FDM the tensile strength of the extruded filaments is much stronger than the fused interfaces between the filaments. If the filaments can be deposited in 3D space instead of being restricted to 2D planes the part strength may be increased substantially (Singamneni et al, 2012). Figure 7 illustrates the difference between an arch-like part made using conventional flat layers and curved layer FDM (CLFDM). The strength of CLFDM parts is increased not only by the orientation of the filaments but by the reduction of the stair step effect, which introduces stress concentrations. CLFDM also allows composite filaments, or other materials, to be used much more effectively. Short fibre reinforcements can be added to the extrudate to increase the tensile strength of the filaments. If the tool path orientation is chosen carefully, this could lead to the extruded reinforcement being in line with the tensile loads, increasing the part strength (Yuan and Gibson, 2008).

An additional benefit of CLFDM is the reduced number of layers required to build thin shells, as shown in figure 7 . This reduces the overall build time as there is less time wasted not extruding between layer movements (Chakraborty et al, 2008).

One disadvantage of CLFDM is that to keep the extruder axis in its ideal position (normal to the tool path) 5 axis control is required. While this will increase the capital cost of the equipment, 5 axis control has been used in other AM technologies such as Laser Engineered Nets Shaping (LENS) (Santos et al, 2005).

\section{HSEF hardware developments}

The fundamental structure of EF hardware has changed little since its conception. Three linear axes are used to move the extrusion head relative to the build platform. The extrusion heads are mounted on linear axes whilst the locomotive force is usually provided by stepper motors. In the late 90's Stratasys increased the build speed of their commercial FDM machines by $500 \%$ by applying their patented MagnaDrive system, which allows the extrusion heads to hang underneath the $x-y$ carriage. The heads are powered independently by electromagnets and separated from the carriage by a frictionless cushion of air (Kreuth et al, 1998). This system reduced the inertia of the extrusion heads and thus allowed higher accelerations. Mechanical improvements of this type are likely to plateau as higher and higher accelerations require more powerful, accurate, rigid and expensive structures and components. The following concepts aim to increase the deposition rate without necessarily increasing the extruder feed rate.

\subsection{Multiple extruders}

One of the factors limiting the size of EF build platforms is the increased build time required to print parts with large cross sectional areas. This is due to the fact the extrusion head needs to travel longer distances to deposit all the material. If the part being built is large enough, or if there are multiple parts on the build platform, then it is possible to use multiple extruders to build the parts simultaneously. Wachsmuth and Bohn, (2008) developed the hardware and software for a multiple extruder system with a detailed examination of the advantages and limitations.

One issue that must be dealt with carefully is the calibration of the extrusion heads with the machine coordinates and build platform. Whilst this will take longer for more extrusion heads it is not likely to be a frequent task. The 
other issue is the possible reduction of strength in the seam produced where the deposition of different extrusion heads meet. Wachsmuth carried out a number of tests to determine how the seam affects the strength of the part. It was found that by overlapping alternate layers of the material from each extruder at least $13 \mathrm{~mm}$ the seam did not negatively impact the parts strength.

The increase in production speed for multiple extruder EF depends on the number of extrusion heads and the suitability of the batch of parts. However it can be said that when manufacturing two identical parts located on opposite sides of the build platform with two extrusion sets (each set consisting of a part and support extruder), the build time will be the same as for one part using conventional FDM.

One could extrapolate this idea to a continuous flow of parts moving along a conveyor with any given number of extrusion heads working simultaneously above the line. The layout of such a facility will resemble that of a fixed automation process, however would still allow customisation at no extra cost. Tool path planning and scheduling will become a big challenge for this type of machine.

\subsection{Variable volumetric deposition extruders.}

Current FDM uses nozzles with small (sub millimetre) orifices. This is necessary in order to accurately position the extrudate where it is needed. The tool path generation software allows for variations in the extruded road width. This is used to deposit narrow roads on the perimeter and wider roads in the interior. Using wider roads for the interior increases the volumetric deposition rate without necessarily increasing the feed rate (although this is also common). A number of factors may limit the volumetric extrusion rate, including; the maximum heating power delivered to the material, the maximum extrusion pressure, the aspect ratio of the roads, the maximum acceleration and the maximum speed of the head relative to the build platform.

The maximum possible variation in road widths is limited by the width to height ratio of the road as shown in Figure 8. Generally the road cannot be taller than it is wide or the road will not fuse with the layer underneath. Also the road cannot be wider than the interface between the nozzle and the road or the material may fill up around the nozzle leaving material above the build layer. Making nozzles with large flat bottom surfaces could extend the maximum width of the roads, however this comes at the cost of interaction with already deposited roads which can lead to missed steps in the motors or movement of the part.

Typically the maximum road width will be reached well before the other factors limiting the volumetric deposition rate such as heating or pressure limits. Therefore it makes sense to provide solutions to this limitation first. One suggested method of overcoming the road width limits is to use variable diameter nozzles (Brooks et al, 2011). These nozzles may be continuously variable, allowing tapered roads, or discretely variable. It was found that for large solid objects, the reduction in build time when using variable diameter nozzles was proportional to the ratio of the maximum and minimum nozzle diameters. Variable diameter nozzles would also be necessary to reach the full potential of the accurate exterior fast interior slicing methods of Sabourin and Bohn, (1996).

Table 1 shows a number of variable diameter nozzle concepts suggested by the authors. These nozzle concepts are currently being evaluated.

The indexing tip concept allows the different nozzle tips to be rotated underneath the heating chamber. The tips could also be configured linearly. While this concept will require more space per extruder, less extruders will be needed. All the nozzle tips will need to be kept at the extrusion temperature to reduce change-over time. Also the extrudate left in each tip after extruding will need to be retracted before the new tip is indexed in. This can be done by reversing the extruder motor to create a vacuum in the tip. Sealing the interface between the tips and the heating chamber will also be a challenge.

The two stage nozzle design is a simple concept for switching between two fixed orifice sizes. The inner nozzle is actuated vertically to block or unblock the larger orifice. While this concept is simple to implement the movement of the inner nozzle displaces material which leads to waste.

The rubber insert concept allows the orifice to be continuously varied by compressing the rubber insert with the pressure ring. Another advantage of this concept is its ability to adjust to changing extrudate properties, as might be the case for functionally graded parts. It is known that some materials are easier to extrude through small nozzles than others. The rubber insert will need to be low friction, conduct heat effectively and withstand high temperatures. Much experimentation will be required to calibrate the location of the pressure ring with the desired 
orifice dimensions For the use of continuously variable nozzles to reach its full potential, new tool path planning algorithms are also needed.

Tseng and Tanaka, (2001) also patented two novel deposition techniques called adaptive filament deposition (AFD) and planar layer deposition (PLD) for the freeform fabrication of metals and ceramics. AFD utilizes a conical spindle inside a deposition head to vary the volumetric flow rate of liquids through the extrusion orifice. After leaving the orifice the liquid jet is cooled by the ambient air temperature and becomes a filament. Retracting the spindle away from the orifice allows higher flow rates which in turn increases the filament size. PLD utilises adjustable planar nozzles and rollers to deposit uniform thickness layers of material. The most notable feature of PLD is the fact that the whole layer can be deposited in one pass. Yang et al, (2000) patented a variable nozzle similar to PLD which allows layers to be deposited in a single pass. Both of these deposition techniques are not well suited to the printing of engineering plastics and require significant alterations to conventional FDM systems. It is also unknown whether these deposition techniques can practically rival the levels of detail achieved by conventional FDM.

\subsection{Multiple material extruders}

One of the advantages of EF is that many different materials can be extruded. Conventional FDM machines can be made to print multi material parts by treating different sub-regions of the part as individual parts in an assembly. In this method the different regions can be printed with separate extruders fed with different build materials.

One important limitation to this method is that to provide functionally graded materials an impractical number of extruders would be needed. Therefore there is a need for extruders capable of depositing materials with variable mixing ratios. This allows functionally graded materials with fewer extruders and the important added benefit of colour control. Corbett and Bowyer, (2012) presented a promising extruder design with a mixing chamber capable of blending multiple colour feed stocks. Real colour printing had previously only been possible using 3D Printing, ink jet printing and LOM.

Stratasys Inc. patented a system for compounding customisable consumable materials in 2010 , although this technology is not yet commercialised.

Further research is required to develop the control software and minimise the amount of purging required between colour changes although this should be made much easier with the adoption of the new standard AM file format AMF.

\subsection{Screw based extruders}

A short mention will be made here on screw extruders. Conventional FDM uses pinch wheels to push polymer filaments through the heating chamber and out the nozzle. The cost of OEM specific plastic filament is often orders of magnitude more expensive than the raw material cost in its powdered or granular form. Extrusion screws can be used to extrude powders as an alternative to filament pinch wheels. Extrusion screws also have the advantage of being able to deposit composite materials such as metal or ceramic powders mixed with binders. Recycling using ground materials is also made easier.

The major disadvantage of screw extruders is the larger size and weight. This reduces the maximum possible acceleration and hence build speed. Filament extruders are likely to remain the dominant extruder type in the near future however screw extruders may find regular use for specialised materials and/or extremely high volume parts.

\section{HSEF material developments}

Material modifications have played a large part in improving the quality and versatility of AM parts. Polymer additives are used to improve the strength, fabrication and appearance of AM parts. Furthermore the unique ability of AM to produce complex multi-material parts and assemblies is one of the most promising attributes of HSEF. HSEF is aimed at consumer and engineering products however a parallel vision for AM in the construction industry has been outlined by Khoshnevis et al (2006) called contour crafting. This section introduces material developments that will extend the potential applications of HSEF. 


\subsection{Ceramics and metals}

Ceramics have unique attributes such as biocompatibility, wear and temperature resistance, electrical and thermal insulation and the piezoelectric effect (Grida and Evans, 2002). Many products would benefit from the addition of ceramic features, however due to manufacturing limitations this is rarely done. HSEF could allow these products to be made more cost effectively.

As ceramics are hard and brittle with very high fusing temperatures it is impossible to use pure ceramic rods as a feed stock for conventional FDM. To get around this a number of extrusion strategies have been developed to use ceramic precursors including; flexible 'green' filaments and ceramic slurries. Once the green part has been fabricated the binder is burnt out and the part is sintered.

Jafari et al, (2000) developed the fused deposition of multiple ceramics (FDMC) system capable of depositing up to four different materials within a given layer. Green ceramic filaments are made by compounding 55-60 vol. percent ceramic powder with ECG-9 binder and then extruded through a multi material extruder. One suggested application is to create piezoelectric ceramic-polymer composites with complex structures to maximise the strain.

Bellini et al, (2005) also developed an ECG-9 binder and PZT composite material but used a screw extruder to process the powder mixture instead of first making green filaments. While much more work is needed to perfect this method it offers greater flexibility in the properties of the composite materials.

Much more research is needed to improve the strength and porosity of ceramic EF parts. A method for selectively sintering ceramic features in multiple material parts is also needed.

Developments in metal EF mirror those found for ceramics but have been driven by the desire for rapid tooling. Metal composite parts would also allow the increase in thermal or electrical conductance to be focussed in localised areas. Wu et al, (2000) describe a process where green filaments loaded with stainless steel powders were printed. The green parts are then sintered in the usual way. Many of the same issues for EF that apply for ceramics apply to metals.

One option currently being investigated by the authors is the possibility of using EF to deposit green state materials which are then consequently sintered or melted with a laser. This could allow the printing of all four main material groups (polymers, metals, ceramics and composites) to be present in each layer.

\subsection{Wood}

A German manufacturer has developed a wood fibre polymer composite filament for EF. While the full composition of the material has not been released, the material is $40 \%$ recycled wood and polymer. The resultant products look and smell like wood with a consistency of medium density fibreboard. By varying the temperature of the extruder and burning the wood to varying degrees the colour of the material can be controlled. This results in a very interesting simulated grain effect (figure 9). The material has proven extremely popular and is an excellent example of on-going material developments.

\subsection{Electronics}

Many of today's consumer products contain electrical components and power sources. Traditionally the manufacture of the electronic components and housing is carried out in separate operations or even factories before being brought together and assembled. These material handling and assembly operations form a significant portion of the products final cost. More importantly, from a design perspective, the standardised electronic components and the design for assembly operations restrict the design of electronic products.

Malone and Lipson, (2008) have developed a number of freeform fabrication techniques for active systems. These include batteries, wiring, strain gauges, electromagnets, actuators, transistors and relays with the aim of eventually building an entire freeform fabricated mobile robot. While the research is in its early stages, they have already demonstrated the potential of these methods. For example they have successfully printed Zinc air batteries with energy and power densities approximately $10 \%$ that of commercial batteries. They have also printed batteries with custom shapes. It is easy to imagine the potential design benefits of freeform batteries. They could fit into unconventional spaces and could form part of the structure of the device itself. 


\section{Future work and opportunities}

AM is no longer an obscure manufacturing method used for prototyping applications. However the economic use of AM for the manufacture of end use components is still restricted to low quantity, low volume parts. Significant collaborative research is needed to develop AM into HSAM. Until now many of the developments leading towards HSAM have been carried out by isolated research groups. There is a substantial opportunity to integrate these advances into a single group of technologies. The following developments for HSEF are required:

- $\quad$ Design automation software to help engineers fully exploit the multi-material advantages of HSEF

- $\quad$ CAD software that simplifies the creation of multi-material mesostructures

- User friendly software for the adaptive layer slicing of multi material parts

- User friendly software for flexible machines capable of multiple build strategies

- Advanced algorithms to determine the optimal build strategies for different parts e.g. flat layer or curved layer FDM

- $\quad$ Cloud based networks allowing vast numbers of uploaded custom designs to be printed in the nearest HSAM facility

- $\quad$ Scheduling algorithms for multi-material, multiple extrusion head production lines

- High speed/acceleration multiple head xy and z carriages

- A variety of multi-material extrusion heads with variable diameter nozzles to cover the main material groups

- $\quad$ Conveyor based build platforms with auto-calibrating extrusion heads and platform positioning

- Image processing software for quality assurance and the online calibration of extrusion rates

- Laser assisted sintering or fusion of green ceramic and metallic features within composite part layers

- Coupling agents and delivery systems for incompatible materials in multi-material parts

- Automated post processing including support removal and surface finishing

- Ecological and sustainability assessments of the new supply chain networks

Figure 10 illustrates the authors' vision of what a future HSAM supply chain might consist of.

\section{Conclusion}

The research developments aggregated in this paper indicate vast improvements on current commercial AM processes are possible. By utilising new software, hardware and materials the production speed and functionality of AM parts may be increased significantly. A new term, High Speed Additive Manufacturing, has been suggested for advanced AM machines capable of competing with traditional manufacturing processes for medium quantity, medium sized parts. These developments include:

- The use of adaptive layer slicing algorithms such as local slicing.

- Tool path planning for multiple materials and functionally graded material parts.

- Collision prevention for multiple extruder machines.

- Design automation for mass customisation and optimisation of multi-material parts.

- $\quad$ Curved layer FDM for thin walled shell like structures and composites.

- Discretely variable and continuously variable volumetric deposition extruders.

- Multiple material extruders with the ability to blend materials with different mixing ratios.

- Screw based extruders for high volume or difficult materials.

- Methods for producing ceramic, metal and wood parts.

- The direct freeform fabrication of active electrical components such as batteries, wiring, strain gauges, electromagnets, actuators, transistors and relays.

Not only can the speed and utility of each extrusion nozzle be improved by software and hardware advances but the technology is easily scalable, both in parallel and in series. The economics of HSAM production machines will become a major issue as parts are built from designs that come from a global design community. Utilisation and availability will have to be closely monitored to match the variable production rates coming from the new designer customer relationships. 
There is also large scope for the hybridisation of AM technologies. By combining the best attributes of laser, inkjet and EF, synergies may be realised that further develop HSAM.

\section{References}

Bernard, A. and A. Fischer (2002). "New Trends in Rapid Product Development." CIRP Annals - Manufacturing Technology 51(2): 635-652.

H. Brooks, M. Lupeanu, T. Abram, (2012). "Production of Personalized Lithophane Lighting Products Using Additive Manufacturing". 4th International Conference on Additive Technologies iCAT 2012. Maribor, Solvenia, $19-21^{\text {st }}$ September. DAAAM International, Vienna: pg 1-10. ISBN 3-901509-92-5.

M.E. Lupeanu, H. Brooks, A.E.W. Rennie, H.K. Celik, C. Neagu, I. Akinci, “Design for Manufacture using Functional Analysis and CAD Mould simulation for Rapid Prototyping and Rapid Tooling", Proceedings of the ASME 2012 11th Biennial Conference on Engineering Systems Design and Analysis,Paper Presented at ESDA2012 CONFERENCE, Iulie 2-4, 2012, Nantes, France, Paper No. ESDA2012-82410 (CD).

Gibson, I., D. Rosen, et al. (2010). Additive Manufactruing Technologies, Springer US.

Hopkinson, N. and P. M. Dickens (2003). Analysis of rapid manufacturing-using layer manufacturing processes for production, Proceedings of the Institution of Mechanical Engineers, Part C : Journal of Mechanical Engineering Science http://www.swetswise.com/link/access_db?issn=0954-4062l.

Crump, S. (1989). Apparatus and method for creating three-dimensional objects. United States, Stratasys, Inc.

Wohlers, T. (2012). Wohlers report 2012. Additive Manufacturing and 3D Printing State of the Industry. ISBN 09754429-8-8.

Tomaz Brajlih, Bogdan Valentan, Joze Balic, Igor Drstvensek, (2011),"Speed and accuracy evaluation of and accuracy evaluation of additive manufacturing machines", Rapid Prototyping Journal, Vol. 17 Iss: 1, pp.64 - 75.

Pulak Mohan Pandey, N. Venkata Reddy, Sanjay G. Dhande, (2003) "Slicing procedures in layered manufacturing: a review", Rapid Prototyping Journal, Vol. 9 Iss: 5, pp.274 - 288.

Pandey, P. M., N. V. Reddy, et al. (2003). "Real time adaptive slicing for fused deposition modelling." International Journal of Machine Tools and Manufacture 43(1): 61-71.

Justin Tyberg and Jan Helge Bohn, "FDM Systems and Local Adaptive Slicing," Materials and Design, vol. 20, no. $2 / 3$, June 1999 , pp. 77-82.

Emmanuel Sabourin, Scott A. Houser, Jan Helge Bøhn, (1997),"Accurate exterior, fast interior layered manufacturing", Rapid Prototyping Journal, Vol. 3 Iss: 2 pp. $44-52$.

W.M. Zhu, K.M. Yu, (2002),"Tool path generation of multi-material assembly for rapid manufacture", Rapid Prototyping Journal, Vol. 8 Iss: 5 pp. $277-283$.

Zhou, M. Y. (2004). "Adaptive slicing of functionally graded material objects for rapid prototyping." The International Journal of Advanced Manufacturing Technology 24(5): 345-352.

Hiller, J., and H. Lipson. 2009. Design Automation for Multi-Material Printing. Solid Freeform Fabrication Symposium (SFF'09), August 3-5, 2009, Austin, Tex.

http://creativemachines.cornell.edu/sites/default/files/SFF09_Hiller2.pdf.

Singamneni, S., A. Roychoudhury, et al. (2012). "Modeling and evaluation of curved layer fused deposition." Journal of Materials Processing Technology 212(1): 27-35.

Yuan, L. (2008). A Preliminary Research on Development of a Fiber-Composite, Curved FDM System. Department of Mechanical Engineering, National University of Singapore. Masters of Engineering. 
Chakraborty, D., B. Aneesh Reddy, et al. (2008). "Extruder path generation for Curved Layer Fused Deposition Modeling." Computer-Aided Design 40(2): 235-243.

Santos, E. C., M. Shiomi, et al. (2006). "Rapid manufacturing of metal components by laser forming." International Journal of Machine Tools and Manufacture 46(12-13): 1459-1468.

Kruth, J. P., M. C. Leu, et al. (1998). "Progress in Additive Manufacturing and Rapid Prototyping." CIRP Annals Manufacturing Technology 47(2): 525-540.

Wachsmuth, J. (2008). Multiple Independant Extrusion Heads for Fused Deposition Modeling. Mechanical Engineering, Virginia Polytechnic Institute and State University. Master of Science.

Brooks, H., Rennie, A., Abram, T., McGovern, J., Caron, F., Variable Fused Deposition Modelling - Advanced Analysis, Concept Design and Tool Path Generation, 5th International Conference on Advanced Research in Virtual and Physical Prototyping, Leira, Portugal, 2011, pp 511-517.

A.A. Tseng, M. Tanaka, (2001) "Advanced deposition techniques for freeform fabrication of metal and ceramic parts", Rapid Prototyping Journal, Vol. 7 Iss: 1, pp.6 - 17.

D. Y. Yang, et al., "Method for Manufacturing Three-Dimensional by Variable Deposition and Apparatus Used Therein.," United States Patent, No. 6,505,089 B1, 2000.

Corbett, J. (2012). Rerap Colour Mixing Project, University of Bath.

Khoshnevis, B., Hwang, D., Yao, K-T. and Yeh, Z. (2006) 'Mega-scale fabrication by contour crafting', Int. J. Industrial and Systems Engineering, Vol. 1, No. 3, pp.301-320.

Grida, I. and J. R. G. Evans (2003). "Extrusion freeforming of ceramics through fine nozzles." Journal of the European Ceramic Society 23(5): 629-635.

M.A. Jafari, W. Han, F. Mohammadi, A. Safari, S.C. Danforth, N. Langrana, (2000) "A novel system for fused deposition of advanced multiple ceramics", Rapid Prototyping Journal, Vol. 6 Iss: 3, pp.161 - 175.

Anna Bellini, Lauren Shor, Selcuk I. Guceri, (2005) "New developments in fused deposition modelling of ceramics", Rapid Prototyping Journal, Vol. 11 Iss: 4, pp.214 - 220.

Wu, G., N. A. Langrana, et al. (2002). "Solid freeform fabrication of metal components using fused deposition of metals." Materials \&amp; Design 23(1): 97-105.

Malone, E. and H. Lipson (2008). Multi-Material Freeform Fabrication of Active Systems. 9th Biennial ASME Conference on Engineering Systems Design and Analysis, ESDA08. Haifa, Israel, ASME.

Figures
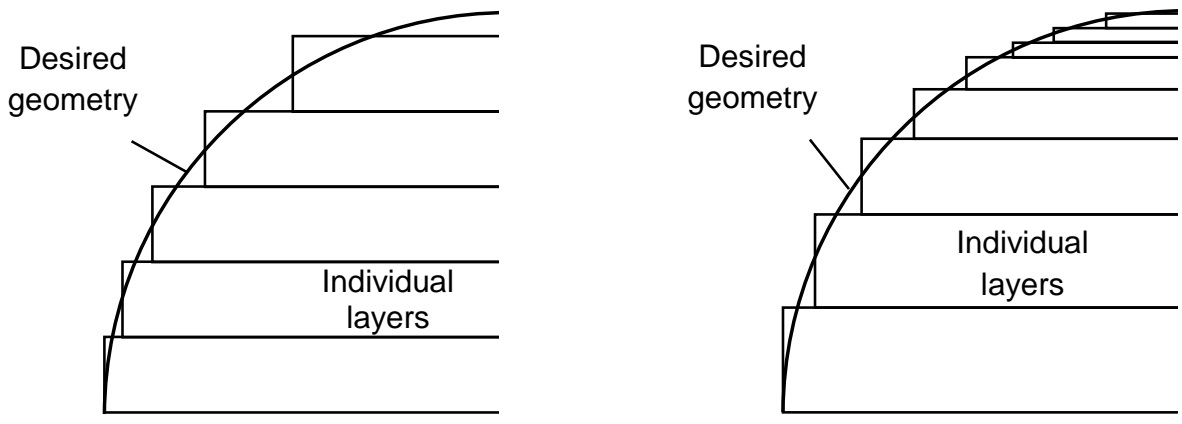

Figure 1. Staircase effect with a) constant layer height and b) variable layer thickness. 


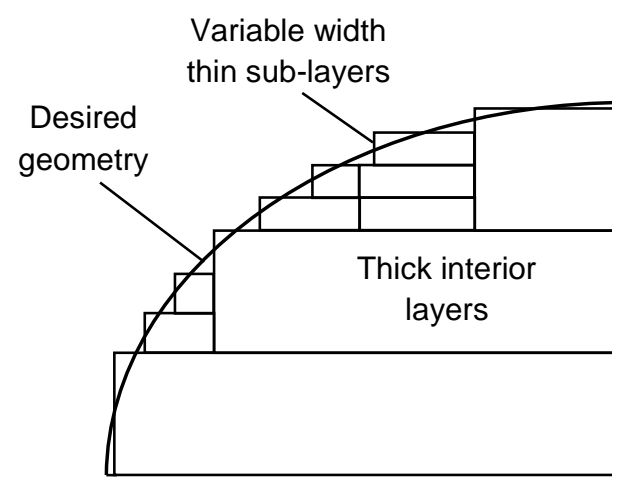

Figure 2. Accurate exterior fast interior layered manufacturing.
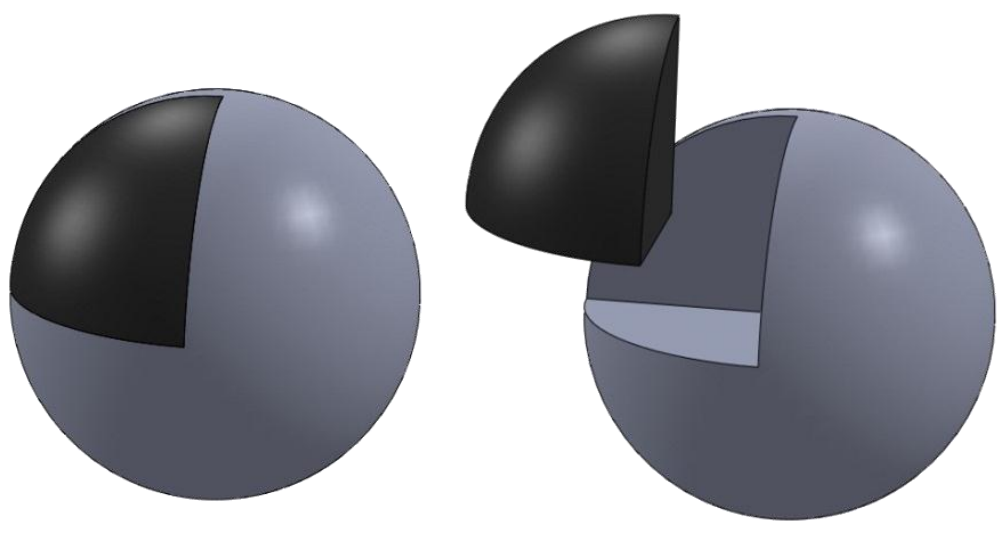

Figure 3. Multiple material part assembly.

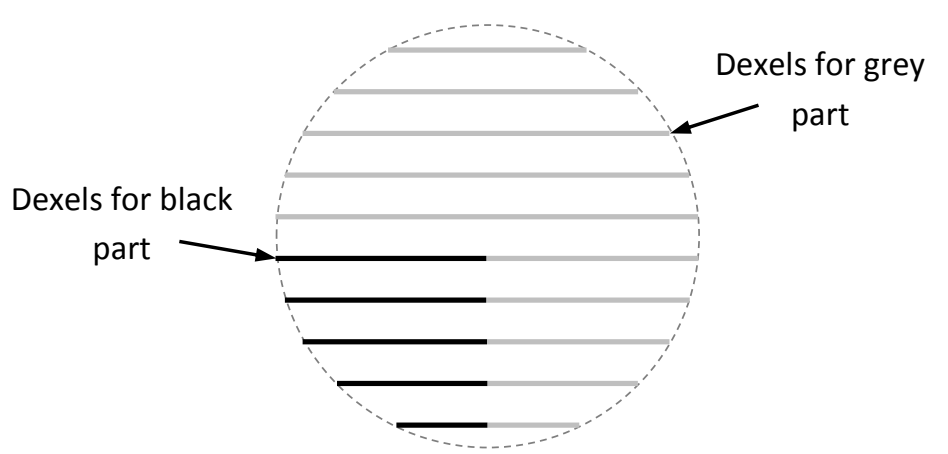

Figure 4. One slice of dexel model showing different materials. 


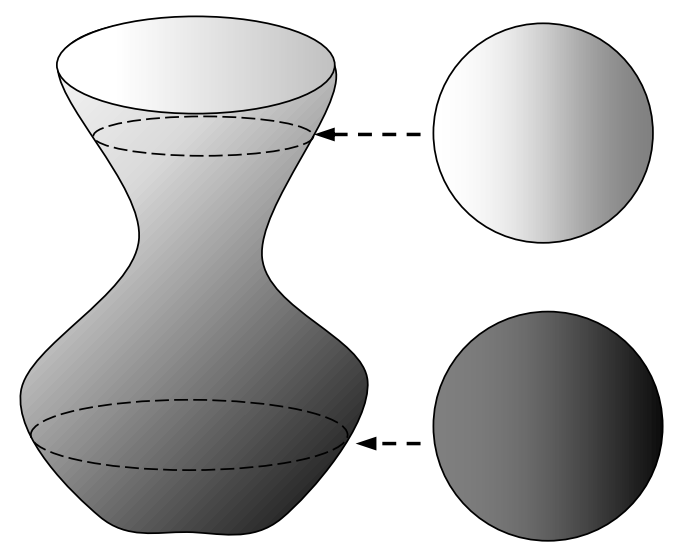

a)

b)

Figure 5. a) A functionally graded part with a white material in the top left corner linearly blending with a black material in the bottom right corner b) cross sections showing planar material composition.

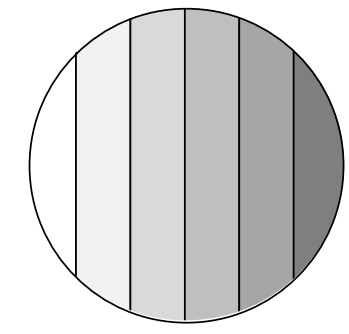

Figure 6. Stepwise approximation of a continuously graded material layer.
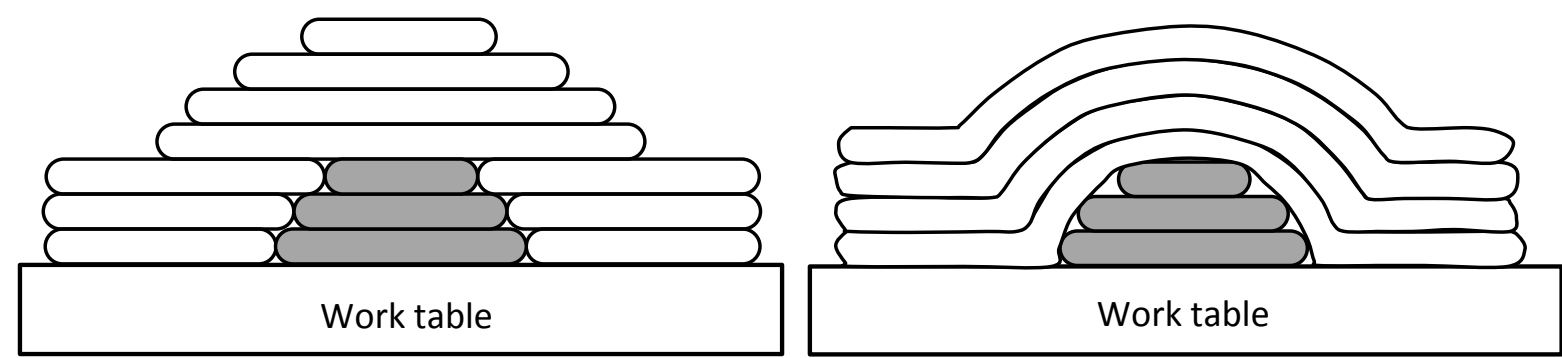

Figure 7. Comparison of the layer structure for conventional FDM and CLFDM. 


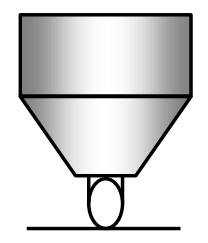

a)

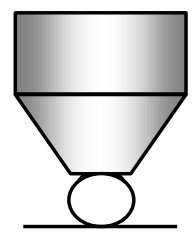

b)

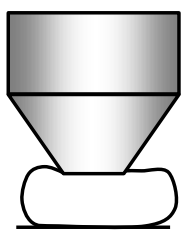

c)

Figure 8. a) road too narrow, b) road with optimal aspect ratio, c) road to wide.

Table 1. Concepts for discrete and continuous variable diameter nozzles.

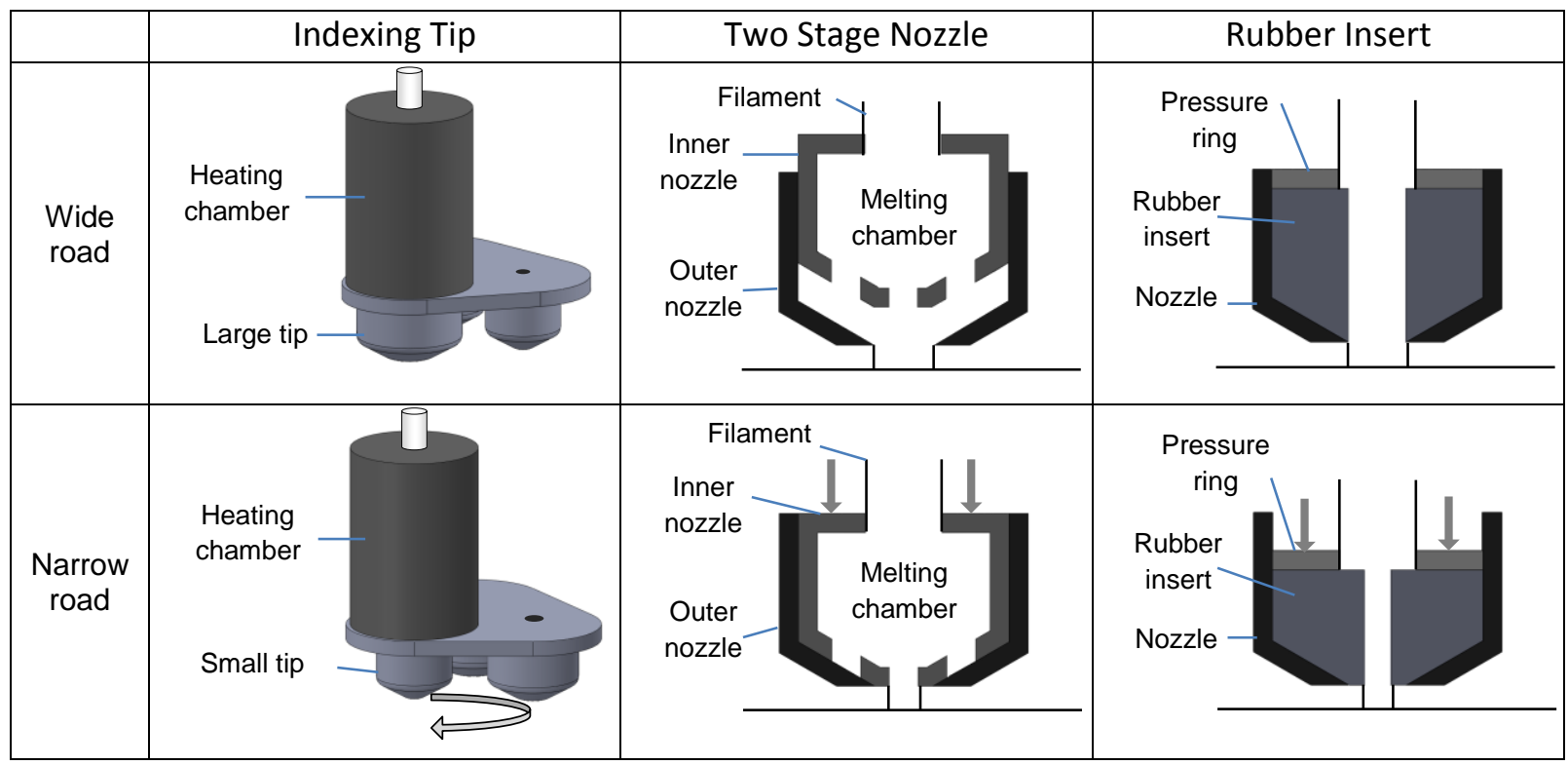

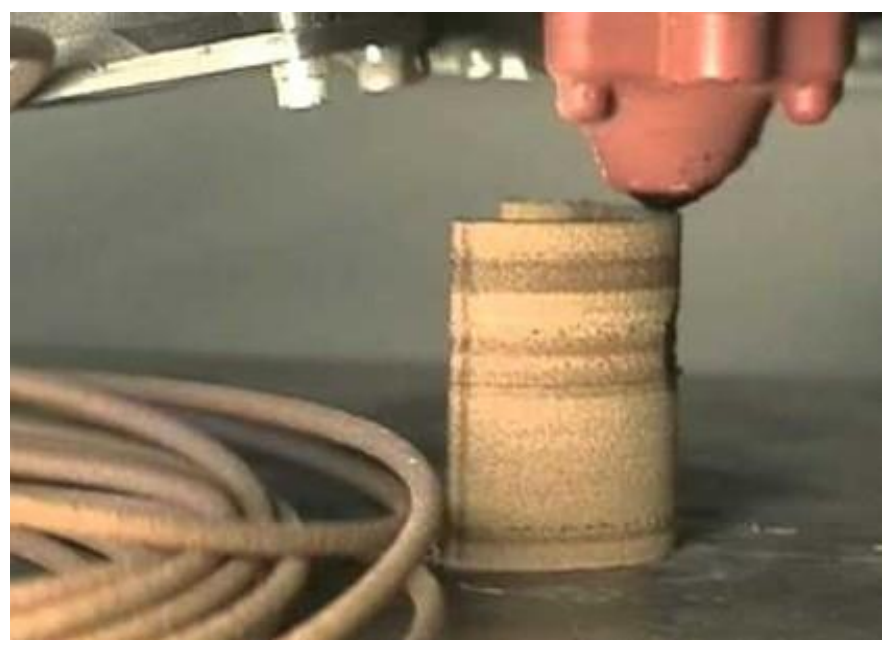

Figure 9. Laywood D3 filament and part being built. 


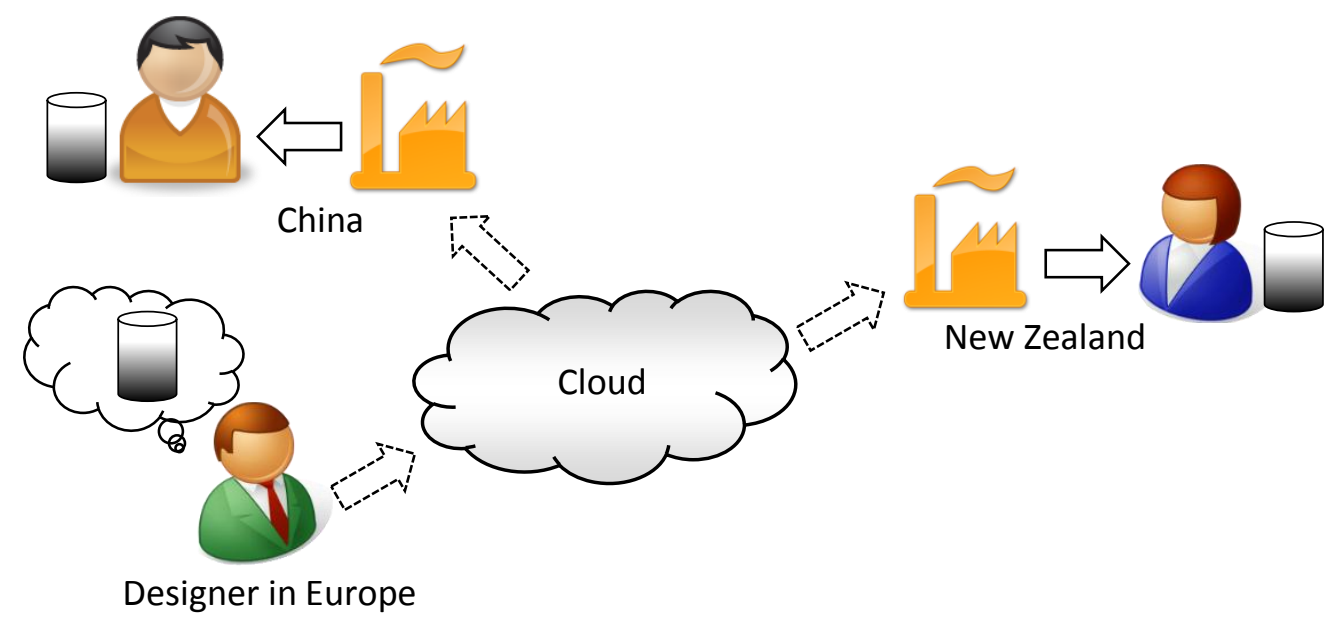

Figure 10. Future HSAM supply chain where manufacture occurs close to the consumer using local materials. 